\title{
Spectroscopy of single-walled carbon nanotubes in aqueous surfactant dispersion
}

\author{
Dania Movia ${ }^{1}$, Elisa Del Canto ${ }^{1}$ and Silvia Giordani ${ }^{* *, 1}$ \\ ${ }^{1}$ School of Chemistry/ Centre for Research on Adaptive Nanostructures and Nanodevices (CRANN), Trinity College Dublin, College \\ Green, Dublin 2, Ireland
}

Received ZZZ, revised ZZZ, accepted ZZZ

Published online ZZZ (Dates will be provided by the publisher.)

PACS 81.07 De, 01.10.Fv, 61.46.Fg, 78.67.Ch

** Corresponding author: e-mail: giordans@tcd.ie, Phone: +00 3531896 1422, Fax: +00 35316712826

\begin{abstract}
Optical studies of single-walled carbon nanotubes (SWNTs) dispersed in aqueous surfactant suspensions have made significant progress since the discovery of their bright band-gap fluorescence in the near-infrared region [1]. In this article we report the systematic investigation of the effect of chemical purification and oxidation on the spectroscopic properties of SWNTs.

The SWNTs samples we prepared differ for the percentage of impurities present, the amount of defects introduced in their graphitic structure and/or the functional groups expressed on their surface.
\end{abstract}

1 Introduction Purification and oxidation of single-walled carbon nanotubes (SWNTs) are key steps in the nanotubes processing in various fields as they increase their solubility, reactivity, and also biocompatibility[2,3]. Oxidation reactions are extensively used for purifying raw SWNTs material from the large amount of impurities that come from their synthesis (such as metal catalysts and fullerenes) [4]; at the same time these procedures generate carboxylic acid $(\mathrm{COOH})$ groups on the nanotubes sidewalls. The use of oxidative protocols is therefore vital to SWNTs application as the $\mathrm{COOH}$ groups are one of the most common anchor groups used for further chemical functionalization of nanotubes sidewalls[2].

One of the most common oxidative protocol is based on a two-step process in which as-produced SWNTs are treated with nitric acid and subsequently with piranha solution $\left(\mathrm{H}_{2} \mathrm{SO}_{4}: \mathrm{H}_{2} \mathrm{O}_{2}\right)$ [5]. The successful formation of $\mathrm{COOH}$ groups on the sidewalls of the SWNTs has been
The generation of carboxylic groups on the nanotubes surface by oxidative treatment was validated by means of TGA and ATR/FT-IR Spectroscopies, as well as Raman Spectroscopy. We have solubilised pristine, purified and oxidized SWNTs in Milli-Q water using sodium dodecylbenzene sulphonate (SDBS). While our Vis-NIR Absorption spectra of purified and oxidized SWNTs dispersions in aqueous SDBS solution show the loss of the resolution of the characteristic van Hove singularities, NIR Photoluminescence spectra are characterized by structured emission peaks.

Copyright line will be provided by the publishe

proved by measurements including Raman and FT-IR Spectroscopies [6].

In this work we report the systematic investigation of the effect of chemical purification and oxidation on the spectroscopic properties of SWNTs. Raw HiPCO SWNTs ( $\boldsymbol{r}$-SWNTs), purified HiPCO SWNTs ( $\boldsymbol{p}$ SWNTs) and oxidized HiPCO SWNTs (o-SWNTS) were characterized by Raman, FT-IR Spectroscopies and TGA. We have solubilised pristine, purified and oxidized SWNTs in Milli-Q water using sodium dodecylbenzene sulphonate (SDBS), a surfactant with polar side chains that creates a solvation shell around the tubes. Vis-NIR Absorption and Emission Spectroscopies were used to analyze the SDBS aqueous dispersions of the different SWNTs. The differences in the spectroscopic results have been evaluated in relation to the purity, oxidation and functionalization grade of the SWNTs utilized. 
2 Experimental Procedure Chemicals were purchased from commercial sources and used as received.

HiPCO SWNTs (purchased from Carbon Nanotechnologies Inc.) were purified and oxidized through a twosteps procedure (Scheme 1) as earlier reported [7].

As-produced raw HiPCO SWNTs (r-SWNTs) were treated with $2.6 \mathrm{M} \mathrm{HNO}_{3}\left(1 \mathrm{~mL} \mathrm{HNO}_{3}\right.$ solution/1 mg nanotubes) at $100{ }^{\circ} \mathrm{C}$ for 48 hours. The suspension was then cooled down to room temperature, filtered through $0.2 \mu \mathrm{m}$ Millipore ${ }^{\circledR}$ isopore filters and washed with distilled water. The purified SWNTs obtained ( $\boldsymbol{p}$-SWNTs) were first dried under vacuum at $60{ }^{\circ} \mathrm{C}$ overnight and then dispersed in a small amount of distilled water by means of sonication for 10 minutes and treated with piranha solution $\left(\mathrm{H}_{2} \mathrm{SO}_{4} / \mathrm{H}_{2} \mathrm{O}_{2} 30 \%\right.$, ratio $4: 1 ; 1 \mathrm{~mL} / 1 \mathrm{mg}$ of nanotubes). The reaction was stirred at $35{ }^{\circ} \mathrm{C}$ for 60 minutes. The oxidation reaction was quenched by diluting the nanotubes suspension with ice and the o-SWNT were filtered through $0.2 \mu \mathrm{m}$ Millipore ${ }^{\mathbb{R}}$ isopore membranes. The product was collected and washed with distilled water until the $\mathrm{pH}$ of the filtrate was neutral. The oxidized SWNTs (o-SWNTs) were finally dried under vacuum at $60^{\circ} \mathrm{C}$ overnight.

Dispersions of $\boldsymbol{r}$-SWNTs, $\boldsymbol{p}$-SWNTs and $\boldsymbol{o}$-SWNTs in aqueous SDBS were prepared in Milli-Q water with an initial nanotubes concentration of $2 \cdot 10^{-2} \mathrm{mg} / \mathrm{ml}$ and with a SWNTs:SDBS mass ratio of 1:25. Nanotubes and SDBS were precisely weighted and dispersed in Milli-Q water by both sonic tip ( 2 minutes) and sonic bath ( 7 hours) treatments. The dispersions were centrifuged at 4,000 RPM for 90 minutes and all optical measurements were carried out on the supernatants in a $1 \mathrm{~cm}$ quartz cells. Absorption Spectroscopy was done on a Perkin Elmer Lambda 35 Absorption Spectrophotometer, while Emission studies were carried out in a L.O.T. ORIEL Nanospectralyzer NS1 at three different excitation wavelengths $(785 \mathrm{~nm}, 683 \mathrm{~nm}$ and $638 \mathrm{~nm})$.Vis Emission studies were carried out on a HORIBA Jobin Yvon Fluorolog-3 Spectrofluorometer at excitation wavelength of $420 \mathrm{~nm}$ with $1 \mathrm{~nm}$ increment, $1 \mathrm{~s}$ of exposure and $14.7 \mathrm{~nm}$ slit width

Infrared spectra were collected on a Perkin Elmer ATR/FT-IR Spectrum 100, while Thermal Gravimetric Analysis was carried out on a TGA Q500 (TA Instruments).Raman spectra were obtained on SWNTs powders using a Renishaw MicroRaman system equipped with a CCD camera and a Leica Microscope. As an excitation source, lasers at $457 \mathrm{~nm}, 514 \mathrm{~nm}$ and $633 \mathrm{~nm}$ with different powers were used.

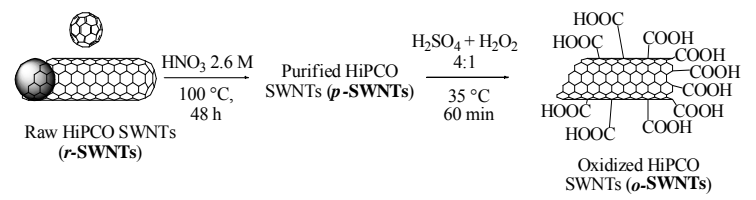

Scheme 1 Purification and oxidation of raw HiPCO SWNTs.

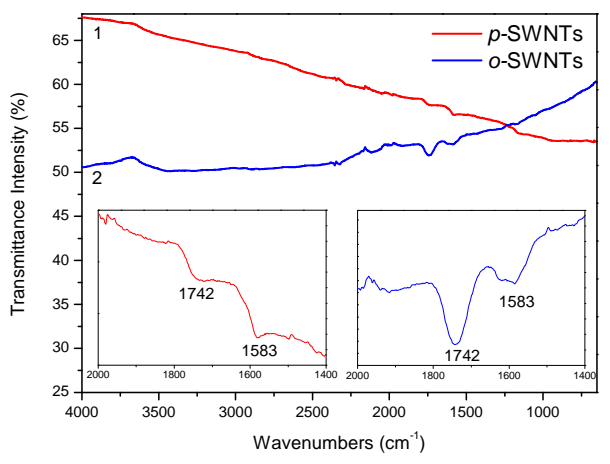

Figure 1 ATR/Fourier transform IR (ATR/FT-IR) spectra of (1) p-SWNTs, and (2) o-SWNTs. In the insets, region 1400-2000 $\mathrm{cm}^{-1}$.

3 Results and Discussion The Thermal Gravimetric Analysis (TGA) in nitrogen of $\boldsymbol{p}$-SWNTs and o-SWNTs show a weight loss at $500^{\circ} \mathrm{C}$ of $11 \%$ and $15 \%$ respectively, as compared to only $4 \%$ of the raw SWNTs ( $\boldsymbol{r}$-SWNTs). This increased weight loss is due to the oxidation of the nanotubes surface and the expression of organic functionalities on tubes surface. The TGA in air of $\boldsymbol{o}$-SWNTs and $\boldsymbol{p}$-SWNTs shows a residue at $900{ }^{\circ} \mathrm{C}$ of approximately $7 \%$ and $4 \%$ respectively, as compared to $26 \%$ for $\boldsymbol{r}$-SWNTs. These results demonstrate that the impurities present in the raw samples are effectively removed by purification and oxidation procedures.

The ATR/FT-IR spectra of nitric acid treated sample ( $\boldsymbol{p}$-SWNTs) and oxidized SWNTs (o-SWNTs) are shown in Figure 1. The low intensity of the peaks in the region of $\mathrm{C}=\mathrm{O}$ stretching transitions (ca. $1730 \mathrm{~cm}^{-1}$ ) in spectrum 1 indicates a low degree of $\mathrm{COOH}$ functionality in the nitric acid treated sample ( $\boldsymbol{p}$-SWNTs). On the other hand, the nanotubes treated with piranha solution (o-SWNTs) show an intense band at $1742 \mathrm{~cm}^{-1}$ (spectrum 2), which has been assigned to the $\mathrm{C}=\mathrm{O}$ stretching transition of the carboxylic acid functions on SWNTs surface [6].

The Raman spectra of $\boldsymbol{p}$-SWNTs and $\boldsymbol{o}$-SWNTs

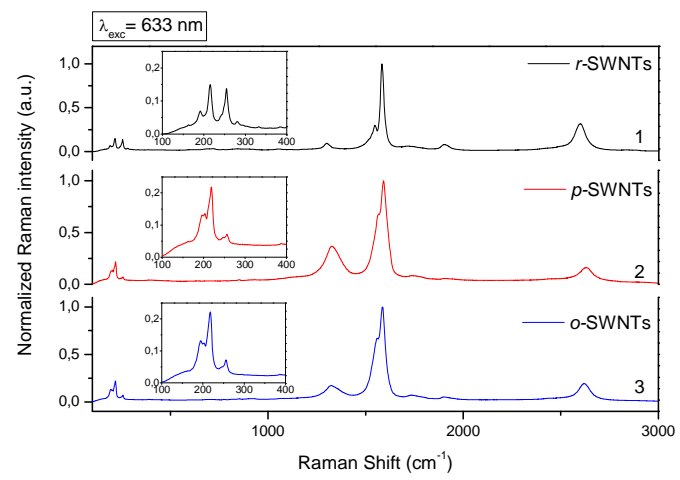

Figure 2 Raman spectra of (1) $\boldsymbol{r}$-SWNTs, (2) $\boldsymbol{p}$-SWNTs, and (3) $\boldsymbol{o}$-SWNTs normalized on the G-band. Laser excitation wavelength is $633 \mathrm{~nm}$. In the insets, radial breathing mode (RBM). 
show high D-bands intensity as compared to $r$-SWNTs (Figure 2). This result consistently indicates an increase of defects density on the surface of purified and oxidized SWNTs. The treatments with nitric acid and piranha solution led to significant changes also in the radial breathing mode (RBM) of the Raman spectra (inserts in Figure 2 ). The bands resonant at higher shifts disappear after nitric acid treatment; this outcome indicates the loss of small diameter SWNTs during the purification and oxidation steps.

NIR photoluminescence studies were performed on $\boldsymbol{r}$-SWNTs, $\boldsymbol{p}$-SWNTs and $\boldsymbol{o}$-SWNTs dispersed in aque-
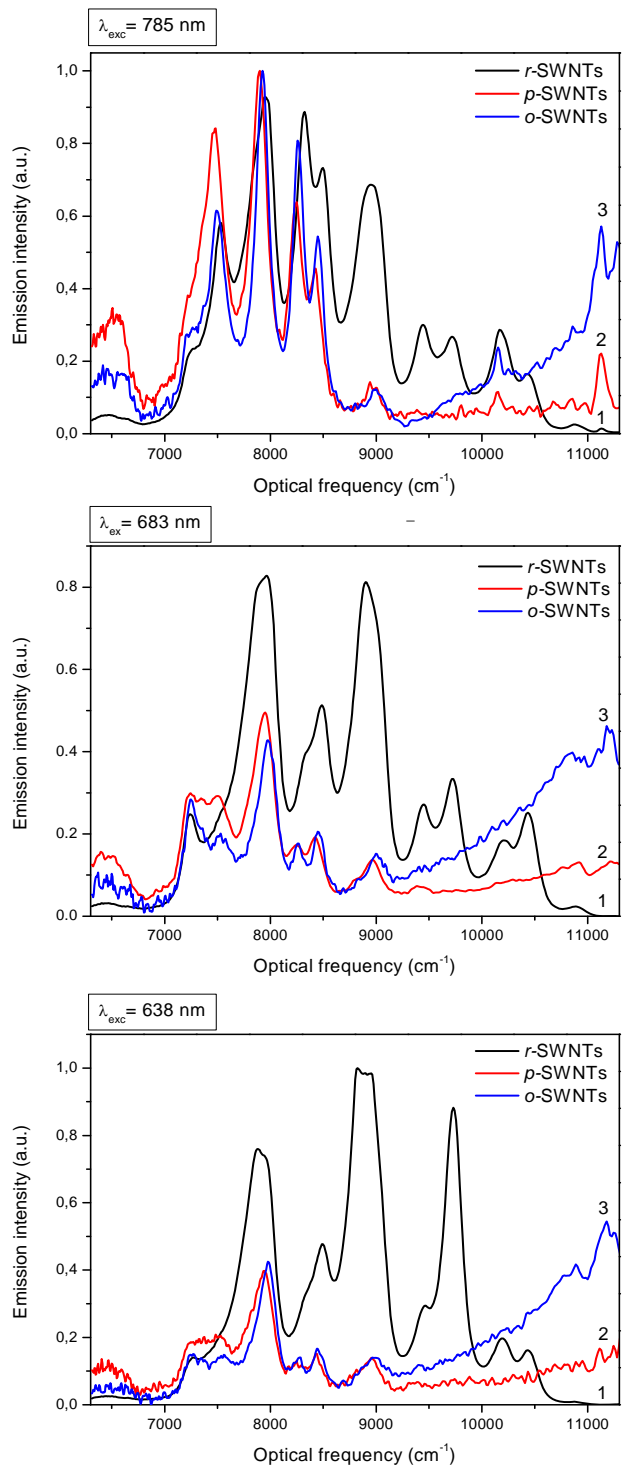

Figure 3 NIR photoluminescence spectra of (1) $r$-SWNTs, (2) $\boldsymbol{p}$-SWNTs, and (2) o-SWNTs. All the samples were dispersed in SDBS aqueous solution and centrifugated at 4,000 RPM for 90 minutes. From the top, laser excitation wavelengths are $785 \mathrm{~nm}$, $683 \mathrm{~nm}$ and $638 \mathrm{~nm}$. [SWNTs $]_{\mathrm{i}}=2 \cdot 10^{-2} \mathrm{mg} / \mathrm{ml}$. ous SDBS dispersion at three different laser excitation wavelengths $(638 \mathrm{~nm}, 683 \mathrm{~nm}$ and $785 \mathrm{~nm})$. The selection of this surfactant was due to the efficacy of SDBS as nanotubes dispersing agent, in order to avoid the quenching due to the intertube interactions in bundled SWNTs. Figure 3 shows the NIR fluorescence spectra $\left(\lambda_{\text {exc }}=785 \mathrm{~nm}, 683 \mathrm{~nm}\right.$, and $\left.638 \mathrm{~nm}\right)$ of SWNTs dispersed in SDBS aqueous solution. The initial concentration of nanotubes is $2 \cdot 10^{-2} \mathrm{mg} / \mathrm{ml}$ and the SWNTs:SDBS mass ratio equal to $1: 25$. The measurements were carried out on supernatants after centrifugation at 4,000 RPM for 90 minutes. It is well-known that chemical functionalization and different degrees of SWNTs oxidation affect the emission behaviour in the visible range: functionalized SWNTs show structured emission in the visible region when excited at short wavelength $[8,9]$. It has been reported that defects on SWNTs surface also affect the nanotubes near-infrared fluorescence. The fluorescence of SWNTs in the NIR region (that generally refers to the definition of band-gap fluorescence [1]) arises from the electronic properties of semiconducting nanotubes. Experimental evidence indicates that this optical property of SWNTs is very sensitive to surface chemistry and defect-producing procedures (such as acid treatment and covalent derivatization of nanotube sidewalls exposed to diazonium salts $[10,11])$ and the perturbation of SWNTs surface by chemical reactions causes the quenching of the nanotubes photoluminescence [10]. When compared to $r$-SWNTs, our purified ( $\boldsymbol{p}$-SWNTs) and oxidised (o-SWNTs) in SDBS aqueous solution were found to display a bright, structured photoluminescence in the NIR region at laser excitation wavelength of $785 \mathrm{~nm}$ (Figure 3). also displayed similar NIR-emission behaviour at this excitation wavelength. Resolved emission features but weaker emission intensities were also registered at excitation wavelengths of $683 \mathrm{~nm}$ and 638 nm (Figure 3). Both $\boldsymbol{p}$-SWNTs and o-SWNTs samples were characterized by a stronger luminescence in the region $10,000 \mathrm{~cm}^{-1}-11,500 \mathrm{~cm}^{-1}$. This effect was earlier described by Lebedkin et al. for acid-treated SWNTs prepared by laser vaporisation method [12]. We suggest that the increased emission in this region of the NIRspectra of $\boldsymbol{p}$-SWNTs and $\boldsymbol{o}$-SWNTs is due to the introduction of defects on nanotubes surface and consequently to their ability of emitting in the visible region.

In order to investigate this effect, Vis- Emission studies were carried out on $\boldsymbol{r}$-SWNTs, $\boldsymbol{p}$-SWNTs and $\boldsymbol{o}$ SWNTs dispersed in SDBS aqueous solution (SWNTs:SDBS mass ratio $=1: 25)$. Figure 4 clearly shows that $\boldsymbol{p}$-SWNTs and $\boldsymbol{o}$-SWNTs were able to emit in the visible region when excited at $420 \mathrm{~nm}$. In particular, the emission intensities resulted higher for these two samples as compared to $\boldsymbol{r}$-SWNTs, confirming the behaviour of the NIR-PL spectra in the range in $10,000 \mathrm{~cm}^{-}$ ${ }^{1}-11,500 \mathrm{~cm}^{-1}$ 
We also investigated the Vis-NIR Absorption Spectroscopy of SWNTs dispersions in SDBS aqueous solution prepared with the same procedure. Vis-NIR absorption spectrum of raw HiPCO SWNTs ( $r$-SWNTs) exhibits well-resolved features (Figure 5), which are characteristic of the first Van Hove $(\mathrm{vH})$ transition of metallic tubes $\left(\mathrm{M}_{11}\right)$ in the region $400-600 \mathrm{~nm}$, the second $\mathrm{vH}$ transition of semiconducting tubes $\left(\mathrm{S}_{22}\right)$ in the 550-900 $\mathrm{nm}$ range, and the first $\mathrm{vH}$ transition $\left(\mathrm{S}_{11}\right)$ of the semiconducting nanotubes with smaller diameters within the 900-1100 nm range [13]. Less-resolved features are detectable in spectra 2 and 3, which correspond to $\boldsymbol{p}$ SWNTs and $\boldsymbol{o}$-SWNTs respectively, as purification and oxidation procedures influence the resolution of the $\mathrm{vH}$ transitions.

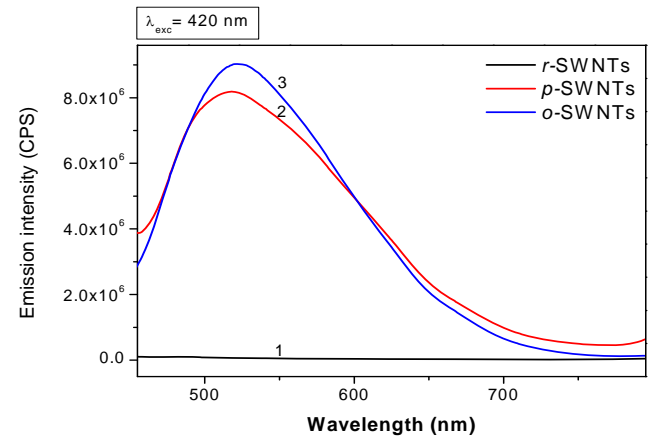

Figure 4 Vis Emission spectra of (1) $r$-SWNTs, (2) $p$ SWNTs, and (3) $o$-SWNTs. All the samples were dispersed in SDBS aqueous solution and centrifuged at 4,000 RPM for $90 \min \left([\mathrm{SWNTs}]_{\mathrm{i}}=2 \cdot 10^{-2} \mathrm{mg} / \mathrm{ml}\right)$.

4 Conclusions In conclusion, we have reported the effects of chemical purification and oxidation on the spectroscopic properties of HiPCO SWNTs. Thermal Gravimetric Analysis show the efficient removal of impurities in our purified and oxidized SWNTs and an increase in the weight loss due to the oxidation of the nanotubes surface. The expression of organic functionalities on the nanotubes surface by the oxidative treatments was confirmed by ATR/FT-IR Spectroscopy. Raman Spectroscopy shows an increase of defects density on the surface of purified and oxidized SWNTs and the loss of small diameter SWNTs after the chemical treatment. Vis-NIR Absorption Spectroscopy of purified and oxidized SWNTs dispersions in aqueous SDBS solution shows the loss of the vH transitions resolution, as compared to $r$-SWNTs. On the other hand, NIR Fluorescence spectra of $\boldsymbol{p}$-SWNTs and $\boldsymbol{o}$-SWNTs aqueous dispersions are characterized by structured features at all excitation wavelengths applied. We are currently further investigating the NIR photoluminescence of oxidized and functionalized SWNTs .

Acknowledgements This work was supported by Science Foundation Ireland (PIYRA 07/YI2/I1052). The authors

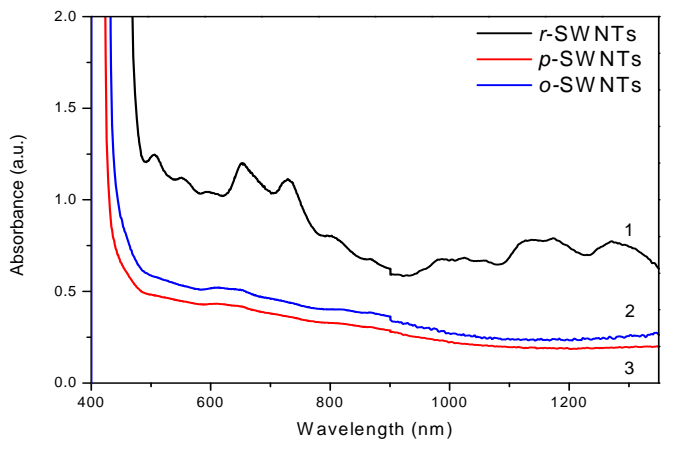

Figure 5 Vis-NIR Absorption spectra of (1) $\boldsymbol{r}$-SWNTs, (2) $\boldsymbol{p}$ SWNTs, and (3) o-SWNTs. All the samples were dispersed in SDBS aqueous solution and centrifuged at 4,000 RPM for 90 $\min \left([\mathrm{SWNTs}]_{\mathrm{i}}=2 \cdot 10^{-2} \mathrm{mg} / \mathrm{ml}\right)$.

wish to acknowledge IRCSET (EMBARK Initiative) and Intel for Postgraduate Research Scholarships to DM and EDC. We also would like to thank Dr. Tatiana Perova, Dr. Manuel Ruther and the support of Trinity College Dublin.

\section{References}

[1] M.J. O'Connell, S.M. Bachilo, C.B. Huffman, V.C. Moore, M.S. Strano, E.H. Haroz, K.L. Rialon, P.J. Boul, W.H. Noon, C. Kittrell, J. Ma, R.H. Hauge, R.B. Weisman, R.E. Smalley, Science. 297 (2002) 593-596.

[2] P. Singh, S. Campidelli, S. Giordani, D. Bonifazi, A. Bianco, M. Prato, Chem. Soc. Rev. 38 (2009) 2214 - 2230.

[3] A. Bianco, K. Kostarelos, C.D. Partidos, M. Prato, Chem. Commun. (2005) 571 - 577.

[4] I.W. Chiang, B.E. Brinson, R.E. Smalley, J.L. Margrave, R.H. Hauge, J. Phys. Chem. B 105 (2001) 1157-1161.

[5] K.J. Ziegler, Z. Gu, H. Peng, E.L. Flor, R.H. Hauge, R.E. Smalley, J. Am. Chem. Soc. 127 (2005) 1541-1547.

[6] U.J. Kim, C.A. Furtado, X. Liu, G. Chen, P.C. Eklund, J. Am. Chem. Soc.. 127 (2005) 15437-15445.

[7] S. Giordani, J.-F. Colomer, F. Cattaruzza, J. Alfonsi, M. Meneghetti, M. Prato, D. Bonifazi, Carbon. 47 (2009) 578588.

[8] Y. Lin, B. Zhou, R.B. Martin, K.B. Henbest, B.A. Harruff, J.E. Riggs, Z.X. Guo, L.F. Allard, Y.P. Sun, J. Phys. Chem. B. 109 (2005) 14779-14782.

[9] D.M. Guldi, M. Holzinger, A. Hirsch, V. Georgakilas, M. Prato, Chem. Comm. (2003) 1130-1131.

[10] L. Cognet, D.A. Tsyboulski, J.D.R. Rocha, C.D. Doyle, J.M. Tour, R.B. Weisman, Science. 316 (2007) 1465 - 1468.

[11] S.M. Bachilo, M.S. Strano, C. Kittrell, R.H. Hauge, R.E. Smalley, R.B. Weisman, Science. 298 (2002) 2361 - 2366.

[12] S. Lebedkin, F. Hennrich, T. Skipa, M.M. Kappes, J. Phys. Chem. B. 107 (2003) 1949-1956.

[13] J.I. Paredes, M. Burghard, Langmuir. 20 (2004) 51495152. 